\title{
Curcumin-loaded sterically stabilized nanodispersion based on non-ionic colloidal system induced by ultrasound and solvent diffusion-evaporation
}

\begin{abstract}
Curcumin has been found to possess significant pharmaceutical activities. However, owing to its low bioavailability, there is a limitation of employing it towards clinical application. In an attempt to surmount this implication, often the choice is designing novel drug delivery systems. Herein, sterically stabilized nanoscale dispersion loaded with curcumin (nanodispersion) based on non-ionic colloidal system has been proposed. In this study, the process conditions were effectively optimized using response surface methodology (RSM) with Boxï Behnken design (BBD). The suggested optimum formulation proved to be an excellent fit to the actual experimental output. STEM images illustrate that the optimal curcumin-loaded nanodispersion has spherical morphology with narrow particle size distribution. Particle size distribution study confirms that the solution $\mathrm{pH}$ does not affect the nanodispersion, and physical stability study shows that the colloidal system is stable over 90 days of storage at ambient conditions. More importantly, controlled release profile was achieved over $72 \mathrm{~h}$ and the in vitro drug release data fit well to Higuchi model (R2=0.9654).
\end{abstract}

Keyword: Anticancer activity; Antioxidants; Colloidal dispersions; Curcumin; Drug release; Green chemistry V; Nanodispersion; Nanotechnology; Optimization; Response surface methodology (RSM); Ultrasound 